\title{
Glow discharge plasma switch controlled by a small magnetic field
}

\author{
J. J. Rocca and K. Floyd ${ }^{\text {a) }}$ \\ Electrical Engineering Department, Colorado State University, Fort Collins, Colorado 80523
}

(Received 12 February 1992; accepted for publication 12 June 1992)

A cold plasma switch scheme with opening and closing capabilities is reported. An externally applied magnetic field causes a low pressure hollow cathode discharge to transition from a low impedance mode sustained by oscillating beam electrons into a virtually collisionless high impedance mode. The impedance of a $10 \mathrm{~A}$ discharge was demonstrated to increase by 20 with the application of a field of $0.018 \mathrm{~T}$.

Switches and modulators are fundamental components in most pulse power applications. Compact high power closing switches have been successfully developed based on discharges which utilize hollow cathodes. ${ }^{1-3}$ These devices have been operated in a glow discharge mode at currents in excess of $100 \mathrm{kA} .{ }^{2}$ It has been recognized that the ability to interrupt a high current hollow cathode discharge (HCD) would have a significant impact in pulse power technology. ${ }^{4,5}$ Magnetic fields are known to affect the impedance of HCDs. ${ }^{6-9}$ A plasma switch based on controlling the plasma density in the axis of a HCD by means of an axial magnetic field has been investigated by Schoenbach et al. ${ }^{4,5}$ In those experiments the applied magnetic field confined the energetic electrons, which normally oscillate radially between the walls of a cylindrical HCD creating an axial region of enhanced ionization, to the periphery of the discharge. An applied magnetic field of $1.2 \mathrm{~T}$ was observed to reduce the plasma density in the axis of the discharge, increasing by a factor of six the impedance of a $10 \mathrm{~A}$ discharge. ${ }^{5}$ In this letter we report a new cold cathode glow discharge plasma switch configuration which requires a significantly smaller magnetic field, of the order of $0.01 \mathrm{~T}$, to modulate the discharge current. In this scheme an externally applied magnetic field causes a HCD to transition from a low impedance regime, sustained by the ionizing collisions of oscillating beam electrons, into a high impedance mode which is virtually collisionless. This is achieved by deflecting to the anode the entire flux of energetic beam electrons. The resulting decrease in the ionization efficiency, which at low pressure can reach two orders of magnitude, quenches the discharge. To implement this scheme a discharge geometry must be selected such that the trajectory of the beam electrons intercepts the anode when a small magnetic field is applied. The discharge geometry shown in Fig. 1, which consists of a pair of parallel plate cathodes placed perpendicular to a pair of parallel plate anodes, is one of the several possible configurations that meet this requirement.

The physical processes that determine the behavior of the discharge are discussed below. It is well known that in cold cathode HCDs the majority of the applied voltage falls in a positive space charge region, the cathode sheath, which develops in front of the cathode. ${ }^{10}$ The rest of the space enclosed by the cathode structure is filled by the

a) On leave from Phillips Laboratory, Kirtland Air Force Base, NM. negative glow, which is practically electric field free and is sustained by the ionization caused by energetic beam electrons accelerated in the cathode sheath. At sufficiently low pressure, the reaching distance of these beam electrons is greater than the distance between the opposite cathode walls, and consequently an oscillatory motion of these ionizing electrons occurs, as schematically illustrated in Fig. 1 (a). The enhanced ionization that results, known as the hollow cathode effect, gives rise to a low impedance discharge which is capable of conducting large currents in a glow mode.

A necessary condition for this glow discharge to be self-sustaining is an electron impact ionization rate sufficiently large to compensate for the loss rate of ions due to diffusion and recombination. Considering that in a low pressure cold cathode HCD the dominant electron emission mechanism from the cathode walls is usually ion bomdardment, and that the secondary electron emission coefficient at common HCD operating voltages is often less than one, ${ }^{11}$ it follows that for the discharge to be selfsustained each beam electron is required to produce multiple electron-ion pairs. Under normal HCD operating conditions (no magnetic field applied) this is achieved by the beam electrons which, oscillating between opposite cathode walls, typically deposit $50 \%$ of their energy into ionizing collisions. ${ }^{12}$ In cases where photoelectron emission from the cathode surface is important, ${ }^{13}$ the rate of ionizing collisions required to sustain the discharge is reduced, but this decrease is offset by an increase in the required number of electron impact excitation collisions. The plasma switch scheme discussed herein is based on drastically reducing the electron ionization and excitation efficiencies by applying a magnetic field such that the trajectory of the beam electrons intercepts the anode, as schematically illustrated in Fig. 1(b). Crucial to this scheme is a low gas pressure such that the mean free path for ionizing collisions is larger than the cathode-anode distance. In this case the beam electrons can be deflected to the anode by the magnetic field in a virtually collisionless trajectory, resulting in a decrease in the ionization efficiency of the order of the ratio between the reaching distance of the beam electrons in the absence of the field to the cathode-anode distance. For example, in a $1600 \mathrm{~V}$ helium discharge at 1.5 Torr with an effective cathode-anode distance of $1 \mathrm{~cm}$ this ratio is calculated to be approximately 100 , allowing for a drastic increase of the discharge impedance. At higher voltages and low gas pressure the ioniza- 


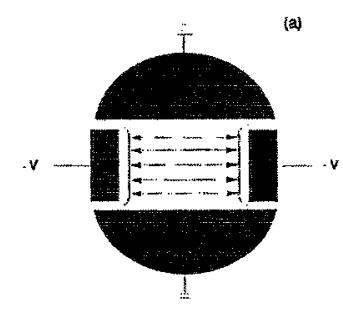

(c)

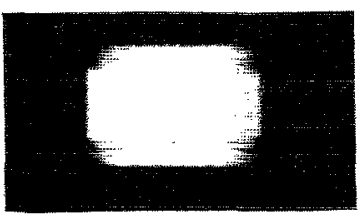

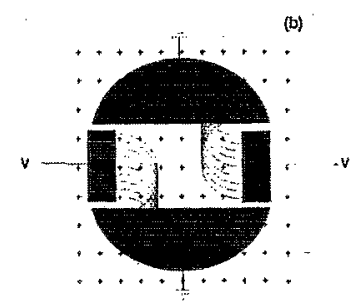

(d)

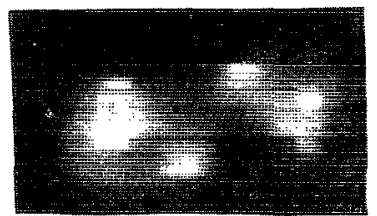

FIG. 1. Cross-sectional view of the HCD schematically illustrating the trajectory of the beam electrons: (a) without, and (b) with magnetic field applied; (c) and (d) are end-on photographs of the glow discharge for $H=0$ and $H=0.018 \mathrm{~T}$, respectively. The helium pressure was 1.2 Torr and the voltage drop across the discharge with the field applied was 1.96 kV.

tion by heavy particles can affect the ionization balance, $^{14,15}$ and should also be considered in designing the device and in selecting the operating pressure.

To demonstrate the principle of the switch discussed above, a HCD of rectangular cross section like that schematically illustrated in Fig. 1 was constructed utilizing brass electrodes and was placed inside an electromagnet. The electrodes were $6 \mathrm{~cm}$ in length with a width of 0.7 and $1.4 \mathrm{~cm}$ for the cathode and anode, respectively. A small gap was left between the electrodes and a surrounding glass tube which served as vacuum container, to confine the discharge to the space enclosed by the electrodes and avoid a discharge from the outside wall of the electrodes. The structure was evacuated utilizing a rotary pump and a needle valve was utilized to establish a controlled helium flow. Direct current and pulse discharges were established by connecting the pairs of electrodes to a dc voltage power supply through a $10 \mathrm{k} \Omega$ ballast resistor, or directly to a pulse generator (Velonex Model 360), respectively. To avoid excessive heating of the discharge structure, which was not refrigerated, the dc discharge experiments were conducted at currents below $40 \mathrm{~mA}$.

Figure 2 is an oscillograph of a magnetic field pulse and the corresponding variation of the discharge current. It illustrates the high degree of modulation of the HCD dc discharge current achieved by turning a magnetic field of $0.018 \mathrm{~T}$ on and off. The dc discharge current, which is initially $30 \mathrm{~mA}$, is observed to decrease in response to the increasing magnetic field strength. The current reduction is approximately proportional to the magnetic field up to 0.01 T. A further increase in the magnetic field strength causes a sudden reduction of the current to a value below $2 \mathrm{~mA}$. Subsequent to the removal of the magnetic field the discharge current recovers to the initial value.

In these experiments the "turn-on" and "turn-off" times of the discharge were limited by the rate of change of the magnetic field, which was slow due to the high induc-

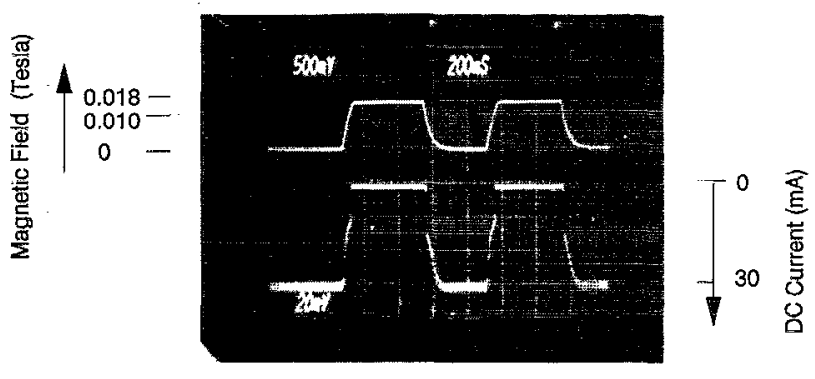

Time $(200 \mathrm{~ms} / \mathrm{div})$

FIG. 2. Modulation of the dc current (upper trace) following the variation of an applied magnetic field (lower trace). The helium pressure was 1.5 Torr and the voltage was $1.5 \mathrm{kV}$.

tance of the solenoid utilized to produce it. No efforts were made to optimize the switching time. Considering that the time necessary for the generation and penetration of the magnetic field into the discharge structure can be minimized utilizing present technology, the time response of the plasma will be limited by the relaxation constant of the plasma density in the negative glow. Potential for short impedance recovery times, of the order of microseconds, arises from the large plasma recombination rates that can result from the very low temperature of the secondary electrons in the negative glow. Although the ionization is primarily produced by energetic electrons, the secondary electrons generated in the process are the most abundant group in the non-Maxwellian electron energy distribution which is characteristic of the negative glow. The low temperature, typically of the order of $0.1 \mathrm{eV}^{16,17}$ results from the peak value at zero energy of the differential cross section for the production of secondary electrons in ionizing electronatom collisions, ${ }^{18}$ and from the field-free characteristics of the negative glow region. Assuming an electron density of $5 \times 10^{13} \mathrm{~cm}^{-3}$, which is within the range for stable HCD operation, the recombination time is calculated to be $1.9 \mu \mathrm{s}$ using the helium ion recombination rate constant given in Ref. 19.

The variation of the dc discharge current as a function of the magnetic field strength is illustrated in Fig. 3(a) for several initial values of the discharge current. In all cases as the magnetic field sirrength is increased an initially linear decrease of the current is followed by a more abrupt drop to a small residual value, in agreement with the oscillograph of Fig. 2. The initial slope corresponds to continuously decreasing values of the gyrotron radius of the beam electrons, and to an increased fraction of the beam electrons intercepting the anode. The more abrupt drop in current occurs when the ionization balance can no longer be maintained throughout the entire negative glow volume. This situation corresponds to the photograph in Fig. 1(d), which shows a faint glow descriptive of the trajectory of energetic electrons for $H=0.018 \mathrm{~T}$.

The effectiveness of the magnetic field in controlling the discharge impedance of the HCD at larger currents was studied utilizing a pulsed discharge. For this purpose a pulse generator, with an output impedance of $12 \Omega$ was 

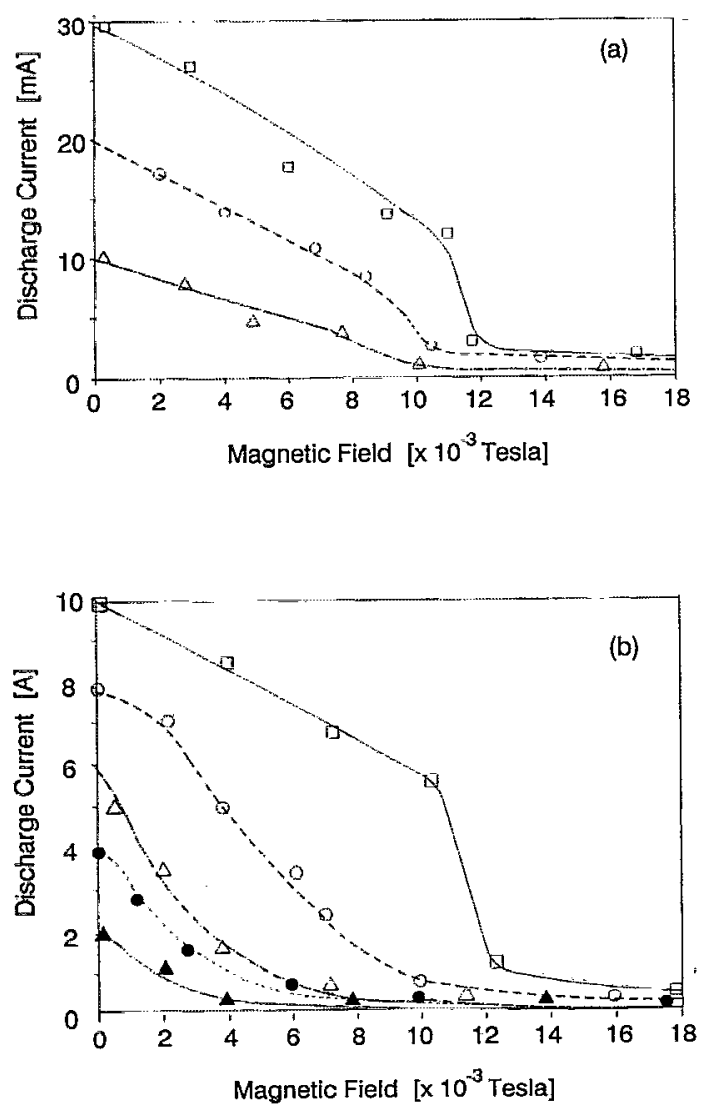

FIG. 3. Variation of the dc (a) and pulsed (b) discharge current as a function of the applied magnetic field for several initial values of the discharge current. The helium pressure was 1.3 and 2.4 Torr, respectively.

used to generate $5 \mu$ s HCD pulses of current up to $10 \mathrm{~A}$ at a repetition rate of approximately $200 \mathrm{~Hz}$. Figure 3(b) shows the variation of the discharge current as a function of the applied cw magnetic field for several initial values of the discharge current. The impedance of a $10 \mathrm{~A}$ discharge was observed to increase by a factor of 20 when a magnetic field of $0.018 \mathrm{~T}$ was applied. Comparison of the low current dc discharge data of Fig. 3(a) with the higher pulsed current data of Fig. 3(b) shows that the applied magnetic field remains effective in quenching the discharge over a current range spanning three orders of magnitude.

Scaling of this concept to larger currents should be possible without a large increase in the required field. Glow to arc transitions absent at the current level of the experiments reported herein can, however, be expected to impose a limitation at very high currents. Therefore a switch based on the principle demonstrated herein is not an alternative to single shot, high power, higher current opening switches such as the Plasma Erosion Opening Switch (PEOS) which can operate at mega-ampere currents. ${ }^{20,21}$ Instead, the concept could possibly be utilized in switches configures to operate at moderate current (kiloamperes) and higher repetition rates.

In conclusion, we have demonstrated a simple technique to switch the impedance of a HCD. An advantage of this plasma switch scheme is the low magnetic field strength required to alter the discharge current. A field of $0.018 \mathrm{~T}$ was shown to increase the impedance of a $10 \mathrm{~A}$ HCD discharge by a factor of 20 . Switching of the operating point of the discharge to the extreme left of Paschen's curve can be achieved in a wide variety of geometries which allow for the entire flux of beam electrons to intercept the anode following a variation of the applied field. The rectangular configuration illustrated in Fig. 1 is but one example of the plasma switch concept described herein.

The authors thank Professor Karl Schoenbach for encouraging discussions and Francisco Gonzalez for the construction of the discharge. The work was supported by an N.S.F Presidential Young Investigators Award (to J. J. Rocca) and of NSF Grant ECS-9013372.

${ }^{1}$ G. Kirkman and M. A. Gundersen, Appl. Phys. Lett. 49, 494 (1986).

${ }^{2}$ R. Frank, E. Boggasch, J. Christiansen, A. Goertler, W. Hartmann, C. Kozlik, C. G. Braun, V. Dominic, M. A. Gundersen, H. Riege, and G. Mechtersheimer, IEEE Trans. Plasma Sci. 16, 317 (1988).

${ }^{3}$ G. Kirkman, W. Hartmann, and M. A. Gundersen, Appl. Phys. Lett. 52, 613 (1988).

${ }^{4}$ M. T. Ngo, K. H. Schoenbach, G. A. Gerdin, and J. H. Lee, IEEE Trans. Plasma Sci. 18, 669 (1990).

${ }^{5}$ K. A. Schoenbach, G. A. Gerdin, T. Tessnow, J. Piekarek, and R. Joshi, 44th Annual Gaseous Electronics Conference, 22-25 October 1991, Albuquerque, NM.

${ }^{6}$ C. Popovich and M. Somesan, Electron. Lett. 1, 31 (1965).

${ }^{7}$ C. Popovich and M. Somesan, Appl. Phys. Lett. 8, 103 (1966).

${ }^{8}$ J. J. Rocca. G. J. Fetzer, and G. J. Collins, Phys. Lett. 84A, 118 (1981). ${ }^{4}$ K. Rozsa, L. Li, G. J. Collins, and P. Apai, Abstracts 44th Annual Gaseous Electronics Conference, 22-25 October 1991, Albuquerque, NM.

${ }^{10} \mathrm{G}$. Francis, Handbuch Der Physik, Gaseous Discharges II (Springer, Berlin, 1956), p. 53.

${ }^{11} \mathrm{G}$. Carter and J. S. Collington, Ion Bombardment of Solids (Elsevier, New York, 1968), p. 22.

${ }^{12}$ G. D. Fetzer and J. J. Rocca, IEEE J. Quantum Electron. (to be published).

${ }^{13}$ P. F. Little and A. von Engle, Proc. R. Soc. Lond. Ser. A 224, 209 (1954).

${ }^{14}$ A. V. Phelps and B. M. Jelenkovic, Phys. Rev. A 38, 2975 (1988).

${ }^{15}$ H. Pak and M. Kushner, J. Appl. Phys. 71, 94 (1992).

${ }^{16} \mathrm{~B}$. Warner, Ph.D. dissertation, University of Colorado, Boulder, CO, 1979.

${ }^{17}$ Z. Yu, J. J. Rocca, G. J. Collins, and C. Y. She, Phys. Lett. 96A, 125 (1983).

${ }^{18}$ L. R. Peterson, Phys. Rev. 187, 105 (1965).

${ }^{19}$ C. B. Collins, A. S. Hicks, W. E. Wells, and R. Burton, Phys. Rev. A 6, 1545 (1973).

${ }^{20}$ J. M. Grossmann, D. Mosher, and P. F. Ottinger, IEEE Trans. Plasma Sci. PS-15, 635 (1987).

${ }^{21}$ J. M. Neri, J. R. Boller, P. F. Ottinger, B. V. Weber, and F. E. Young, Appl. Phys. Lett. 50, 1331 (1987). 